\title{
Direct and indirect gene diagnosis of hemophilia A pedigrees in the Chinese population
}

\author{
YING CAO $^{1}$, LUXI SHEN ${ }^{2}$, RONG HUANG ${ }^{1}$, LINGCONG YANG ${ }^{1}$, ZHUANYUN DU $^{1}$, \\ HUA MA $^{1}$, TIANJIN ZHENG ${ }^{3}$, HAIHUA GU ${ }^{1}$ and HONGZHI LI ${ }^{1}$ \\ ${ }^{1}$ The Zhejiang Provincial Key Laboratory of Medical Genetics, School of Life Sciences, Wenzhou Medical \\ University, Wenzhou, Zhejiang 325035; ${ }^{2}$ Department of Graduate Students, Xuanwu Hospital, Capital \\ Medical University, Beijing 100053; ${ }^{3}$ Department of Technical Guidance, Family Planning Publicity \\ and Technical Guidance Station of Wenzhou City, Wenzhou, Zhejiang 325000, P.R. China
}

Received October 20, 2016; Accepted June 16, 2017

DOI: $10.3892 / \mathrm{mmr} .2017 .7263$

\begin{abstract}
Hemophilia A (HA) is an X-linked recessive hereditary disorder caused by defects in the coagulation factor VIII (FVIII) gene. In order to diagnose patients with presymptomatic HA and carriers, the present study conducted direct gene diagnosis for the common abnormalities in FVIII and subsequently performed indirect gene diagnosis for the other abnormalities in FVIII for Chinese HA pedigrees. Direct gene diagnosis was performed in $10 \mathrm{HA}$ pedigrees using inverse shifting-polymerase chain reaction to detect intron 22 inversion (inv22), intron 22 deletion, intron 22 duplication and inv1 of FVIII. Pedigrees with no detected mutations were further analyzed using indirect genetic diagnosis (haplotype linkage analysis), where the genetic markers of FVIII included one variable number of tandem repeat, seven short tandem repeats and three restriction fragment length polymorphisms. The results of three pedigrees were taken as examples. Pedigree 1 underwent direct gene diagnosis, which demonstrated that the proband was inv22 distal pattern hemophiliac and the mother was an inv22 distal pattern carrier. The other two pedigrees were subjected to indirect gene diagnosis. In pedigree 2 , the detection of DXS52, 13(CA) n, DXS9901(GT) n, intron (int)18, int 19 and int 22 confirmed the proband's baby brother was normal, the proband's maternal aunt was a carrier and her baby son was normal. Detection of DXS9901(GT)n, int18, int19 and int22 in pedigree 3 demonstrated that the proband's maternal grandmother was not a carrier. As the maternal grandfather
\end{abstract}

Correspondence to: Professor Hongzhi Li or Professor Haihua Gu, The Zhejiang Provincial Key Laboratory of Medical Genetics, School of Life Sciences, Wenzhou Medical University, Tongde Building, North Zhongxin Road, Chashan University Park, Wenzhou, Zhejiang 325035, P.R. China

E-mail:wzmclhz@aliyun.com

E-mail: haihuagu@gmail.com

Key words: hemophilia A, coagulation factor VIII gene, direct gene diagnosis, indirect gene diagnosis, haplotype linkage analysis was not affected by the disease, it was deduced that a mutation of FVIII occurred in the proband's mother. The combination of direct and indirect gene diagnoses provides reliable evidence for the use of genetic counseling in HA pedigrees, particularly for screening presymptomatic males and female carriers with normal offspring.

\section{Introduction}

Hemophilia A is a hemorrhagic disease occurred much more frequently in males than in females, since it is an X-linked recessive hereditary disorder caused by diverse mutations in the coagulation factor VIII (FVIII) gene that affects 1 in 5,000 male newborns (1). Patients are primarily infused with FVIII products for hemorrhage prophylaxis and there is currently no radical cure. FVIII gene mapping to Xq28 is $186 \mathrm{~kb}$ long and consists of 26 exons and 25 introns (2). A total of $20 \%$ of HA cases have a genetic etiology of intron 22 inversion (inv22) in the FVIII gene (3), which can be detected via direct gene diagnosis. However, the majority of patients with HA harbor different FVIII mutations, of which there are $>1,000$ types, thus direct gene diagnosis is difficult to perform (4). Consequently, linkage analysis using closely-linked genetic markers (extragenic and intragenic) of the FVIII gene, has emerged as a principal method for detecting carriers. Genetic diagnosis of HA carriers is one of the most effective ways to reduce the birth numbers of newborns with HA. Currently the most widely used markers are variable number of tandem repeats (VNTRs), short tandem repeats (STRs) and restriction fragment length polymorphisms (RFLP) (5). However, the linkage analysis method has limitations as families lacking heterozygous genetic markers are not suitable for this type of analysis (5). Recombination between the genetic marker and mutation locus may also lead to misdiagnosis (5). Therefore, in the present study, direct diagnosis, using inverse shifting-polymerase chain reaction (IS-PCR) to detect inv22 and inv1, was performed in combination with indirect gene diagnosis methods, using 11 genetic marker loci of VNTR, STR and RFLP for haplotype linkage analysis, for the genetic diagnosis of HA. The aim of the present study is to provide reliable evidence for genetic counseling for HA 
pedigrees, particularly for screening presymptomatic males and female carriers with normal or no offspring, which may facilitate earlier treatments and disease prevention.

\section{Materials and methods}

Samples and DNA extraction. The present study was approved by the Ethics Committee of Wenzhou Medical University (Zhejiang, China) and written informed consent was obtained for all participants. Investigations were performed in 10 HA pedigrees recruited by the Family Planning Publicity and Technical Guidance Station of Wenzhou City between January 2008 and December 2014 in Wenzhou, China. All ten patients with HA were males aged 4-12 years old with a history of bleeding; HA diagnoses were verified by confirming each patient exhibited very low levels of Factor VIII. Blood samples were obtained from the ten probands and other 42 members of the pedigrees. Genomic DNA was extracted using the standard phenol chloroform method (6).

Direct gene diagnosis. The present study developed the direct gene diagnosis methodology using IS-PCR to detect inv22 and inv1. In addition, supplementary experiments were performed to detect intron 22 deletion (del22) and intron 22 duplication (dup22) in order to avoid misdiagnosis for inv22. The following primers were used: For inv22, the ID, $1 \mathrm{U}, 2 \mathrm{U}$ and $3 \mathrm{U}$ primers were used; for del22 and dup22, the ED, 1U, 2U and $3 \mathrm{U}$ primers were used; and for inv1, the1-ID, $1-I U$ and 1-ED primers were used. The sequences of these 8 primers are listed in Table I (7). The DNA samples were digested using $B c l$ I enzyme. The purified products were then ligated with T4 DNA ligase and purified. Finally, IS-PCR amplification $\left(95^{\circ} \mathrm{C}\right.$ for $30 \mathrm{sec}, 60^{\circ} \mathrm{C}$ for $60 \mathrm{sec}, 72^{\circ} \mathrm{C}$ for $90 \mathrm{sec} ; 35$ cycles) was performed. The products were run on a $1 \%$ agarose gel with ethidium bromide, and the image was reversed. The diagnoses were made based on the different fragment lengths of the IS-PCR products for FVIII, which are presented in Table II, and the experiments were repeated three times.

Indirect gene diagnosis. As different pedigrees may have different effective genetic marker loci, the present study used $\leq 11$ genetic marker loci for linkage analysis of HA pedigrees. These sites included one FVIII extragenic VNTR site (DXS52), three FVIII extragenic STR sites [DXS15(CA)n, DXS9901(GT)n and DXS1073(GT)n], four FVIII intragenic STR sites [1(GT)n, 13(CA)n, 22(GT)n(AG)n and 24(GT) n] and three FVIII intragenic RFLP sites [intron (int)18, int19 and int22]. The distribution of these genetic marker loci inside or outside the FVIII gene is shown in Fig. 1. The present study used PCR to amplify the target fragments of VNTR, STR or RFLP loci, and then performed analysis of the polymorphisms. VNTR polymorphisms were reflected by the PCR fragment lengths identified via $1 \%$ agarose gel electrophoresis with ethidium bromide, and the image was reversed. STR polymorphisms were reflected by the PCR fragment lengths observed following 6\% PAGE with silver staining and capillary electrophoresis. RFLPs were reflected by the length of enzyme-digested PCR fragments identified on $6 \%$ PAGE gels with silver staining. Annealing
Table I. Sequences of the primers used for inverse shifting-polymerase chain reaction.

\begin{tabular}{ll}
\hline Primer & \multicolumn{1}{c}{ Sequence $\left(5^{\prime}-3^{\prime}\right)$} \\
\hline ID & ACATACGGTTTAGTCACAAGT \\
ED & TCCAGTCACTTAGGCTCAG \\
$1 \mathrm{U}$ & CCTTTCAACTCCATCTCCAT \\
$2 \mathrm{U}$ & ACGTGTCTTTTGGAGAAGTC \\
$3 \mathrm{U}$ & CTCACATTGTGTTCTTGTAGTC \\
$1-\mathrm{IU}$ & GCCGATTGCTTATTTATATC \\
$1-\mathrm{ID}$ & TCTGCAACTGGTACTCATC \\
$1-\mathrm{ED}$ & GCCTTTACAATCCAACACT \\
\hline
\end{tabular}

temperatures, primer sequences and PCR product fragment sizes of the genetic marker loci are summarized in Table III (8-11). All of the PCR amplification except for DXS52 was carried out according to the following condition for 35 cycles: Denaturation at $95^{\circ} \mathrm{C}$ for $45 \mathrm{sec}$; annealing at the temperature indicated in Table III for $45 \mathrm{sec}$; extension at $72^{\circ} \mathrm{C}$ for $45 \mathrm{sec}$. For the DXS52 amplification, PCR was carried out for 26 cycles: $95^{\circ} \mathrm{C}$ for $40 \mathrm{sec}, 60^{\circ} \mathrm{C}$ for $40 \mathrm{sec}$, $72^{\circ} \mathrm{C}$ for $90 \mathrm{sec}$. The PCR amplification was carried out using high fidelity PCR Mix (Vazyme, Piscataway, NJ, USA). The diagnoses made based on the size of enzyme-digested products are presented in Table IV, and the experiments were repeated three times.

\section{Results}

Direct gene diagnosis of HA pedigree 1. DNA samples from 10 probands were analyzed by IS-PCR in order to detect inv22. Only one proband (pedigree 1) harbored the inv22 mutation. Subsequently, supplementary experiments were performed to confirm that it was inv22 and not del22 or dup22. Electrophoresis of the IS-PCR products for inv22 revealed that the proband was a distal inv22 homozygote (Fig. 2A). The proband's mother was a distal inv22 heterozygote and the father was normal. The results of electrophoresis of the IS-PCR products for del22 or dup22 revealed that neither the proband, nor the proband's mother or father had del22 or dup22 mutations (Fig. 2B). In addition, IS-PCR was performed in order to detect inv1 in the other 9 probands; however, inv1 was not detected in any of these patients.

Indirect gene diagnosis of HA pedigree 2 and 3. In the present study, 11 genetic marker loci were used for linkage analysis of the other nine pedigrees without inv 22 and inv1; there were $\geq 4$ genetic marker loci that could provide effective information for each pedigree. As the different families had different effective genetic marker loci, the present study presents two pedigrees as examples to present the results of indirect gene diagnosis analysis in the present study.

Pedigree 2. Detection of the 11 genetic marker loci for pedigree 2 , identified six marker loci that were heterozygous and could provide effective linkage information, including one VNTR locus (DXS52), two STR loci [13(CA)n and 
Table II. Diagnoses made based on the different fragment sizes of the inverse shifting-polymerase chain reaction products for the coagulation factor VIII gene.

\begin{tabular}{lcc}
\hline & & Fragment size, bp \\
\cline { 2 - 3 } Diagnosis & Inv22 test & Del22 and dup22 test \\
\hline Male & & \\
Normal & 487 & 457 and 405 \\
Inv-1 & & \\
Inv22-1(distal) & 333 & 559 and 457 \\
Inv22-2(proximal) & 385 & 559 and 405 \\
Dup22 & 487 & 459,457 and 405 \\
Del22-1 & 333 & 405 \\
Del22-2 & 385 & 224 \\
Female & & 457 and 405 \\
Normal homozygote & 487 & \\
Inv-1 heterozygote & & 559,457 and 405 \\
Inv22-1 heterozygote & 487 and 333 & 559,457 and 405 \\
Inv22-2 heterozygote & 487 and 385 & 559,457 and 405 \\
Dup22 heterozygote & 487 & 457 and 405 \\
Del22-1 heterozygote & 487 and 333 & 457 and 405 \\
Del22-2 heterozygote & 487 and 385 & 304 and 224 \\
\hline
\end{tabular}

Inv, intron inversion; del, intron deletion; dup, intron duplication.

Table III. Annealing temperature, primer sequences and fragment size for the polymerase chain reaction of genetic markers.

\begin{tabular}{|c|c|c|c|c|c|}
\hline $\begin{array}{l}\text { Author, } \\
\text { year }\end{array}$ & $\begin{array}{l}\text { Genetic } \\
\text { marker }\end{array}$ & $\begin{array}{l}\text { Annealing } \\
\text { temperature }\end{array}$ & $\begin{array}{l}\text { Primer sequence } \\
\text { and label }\left(5^{\prime}-3^{\prime}\right)\end{array}$ & $\begin{array}{l}\text { Fragment } \\
\text { size, bp }\end{array}$ & (Refs.) \\
\hline Yan et al, 2011 & DXS52 & $60^{\circ} \mathrm{C}$ & GGCATGTCATCACTTCTCTCATGTT & $700-3,000$ & $(8,9)$ \\
\hline Richards et al, 1991 & & & CACCACTGCCCTCACGTCACTT & & \\
\hline Fang et al, 2006 & DXS15(CA)n & $60^{\circ} \mathrm{C}$ & $\begin{array}{l}\text { AGCACATGGTATAATGAACCTCCACG } \\
\text { (FAM)-CAGTGTGAGTAGCATGCTAGCAT } \\
\text { TTG }\end{array}$ & $148-168$ & $(10)$ \\
\hline Fang et al, 2006 & DXS9901(GT)n & $62^{\circ} \mathrm{C}$ & $\begin{array}{l}\text { GACCAGTCCTCCCTTCTGTT } \\
\text { (FAM)-GTGTGGAGTGAAAGGGACAG }\end{array}$ & $186-214$ & $(10)$ \\
\hline Fang et al, 2006 & DXS1073(GT)n & $60^{\circ} \mathrm{C}$ & $\begin{array}{l}\text { ATGCCCTCTCCGAGTTATTACA } \\
\text { (FAM)-ATTGGTGGCCTTTGAAACAC }\end{array}$ & $122-142$ & $(10)$ \\
\hline Liang et al, 2009 & 1(GT)n & $55^{\circ} \mathrm{C}$ & $\begin{array}{l}\text { GGTTCTCCAATGAAACAGATCC } \\
\text { CCTTGGACATAAGCATTCTTGG }\end{array}$ & $120-132$ & $(11)$ \\
\hline Liang et al, 2009 & $13(\mathrm{CA}) \mathrm{n}$ & $45^{\circ} \mathrm{C}$ & $\begin{array}{l}\text { GTTTCTTTGCATTCAACTGTACA } \\
\text { TAATGTATCTT } \\
\text { CCAAATTACAGATTGAATAAGCCTAG }\end{array}$ & $146-172$ & (11) \\
\hline Liang et al, 2009 & $22(\mathrm{GT}) \mathrm{n}(\mathrm{AG}) \mathrm{n}$ & $50^{\circ} \mathrm{C}$ & $\begin{array}{l}\text { GTTTCTTATTAATGCCCACATTATAGACT } \\
\text { CTC } \\
\text { AATAAGACCCTTAGCTGTTTCAT }\end{array}$ & $207-219$ & $(11)$ \\
\hline \multirow[t]{4}{*}{ Liang et al, 2009} & 24(GT)n & $58^{\circ} \mathrm{C}$ & $\begin{array}{l}\text { GCCTGGACTACAGAGGGAGAC } \\
\text { AAATTTTCACACGCACACCTG }\end{array}$ & $182-198$ & (11) \\
\hline & Intron 18 & $58^{\circ} \mathrm{C}$ & $\begin{array}{l}\text { TTCATTTCAGTGGACATGTG } \\
\text { CCTATGGGATTTGAGATGGT }\end{array}$ & 374 & \\
\hline & Intron 19 & $55^{\circ} \mathrm{C}$ & $\begin{array}{l}\text { TTGGCGAGCATCTACATGCT } \\
\text { CTAATGTGTCCAGAAGCCAT }\end{array}$ & 702 & \\
\hline & Intron 22 & $60^{\circ} \mathrm{C}$ & $\begin{array}{l}\text { CACGAGCTCTCCATCTGAACATG } \\
\text { GGGCTGCAGGGGGGGGGGACAACAG }\end{array}$ & 96 & \\
\hline
\end{tabular}




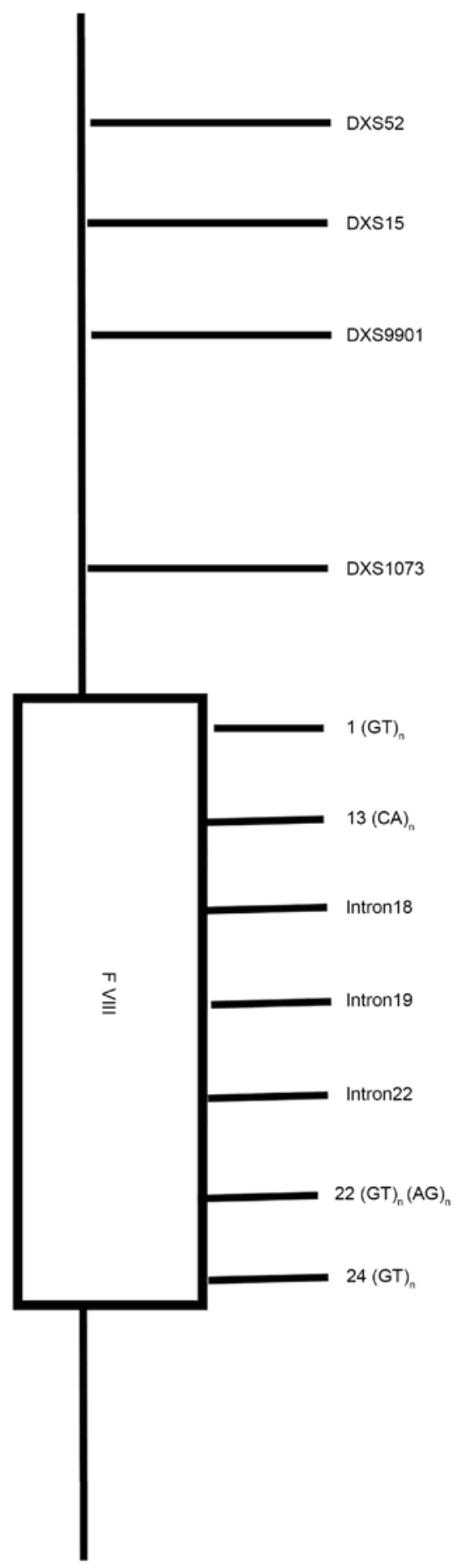

Figure 1. Distribution of the genetic marker loci inside and outside of the FVIII gene that were used in the present study. FVIII, coagulation factor VIII.

DXS9901(GT)n] and three RFLP loci (int18, int19 and int22). The genogram and the electrophoretogram of the PCR products of pedigree 2 are presented in Fig. 3.

The results of the DXS52, DXS9901(GT)n and 13(CA) $\mathrm{n}$ loci (Fig. 3B-D) demonstrated that the band of the proband (III1) was in the same position as the proband's mother's (II3) lower band, indicating that the $\mathrm{X}$ chromosome was pathogenic and the mother was a carrier. The band of the proband's baby brother (III2) was in the same position as the mother's upper band, indicating his X chromosome was normal. The pathogenic X chromosome of the proband's maternal aunt (II5) and
Table IV. Diagnoses made based on the size of the enzyme-digested products.

\begin{tabular}{llcc}
\hline RFLP loci & Enzyme & $\begin{array}{c}\text { Allele } \\
\text { negative, bp }\end{array}$ & $\begin{array}{c}\text { Allele } \\
\text { positive, bp }\end{array}$ \\
\hline Intron 18 & BclI & 374 & 211 and 163 \\
Intron 19 & HindIII & 467 and 235 & 467,154 and 81 \\
Intron 22 & XbaI & 96 & 68 and 28 \\
\hline
\end{tabular}

RFLP, restriction fragment length polymorphisms.

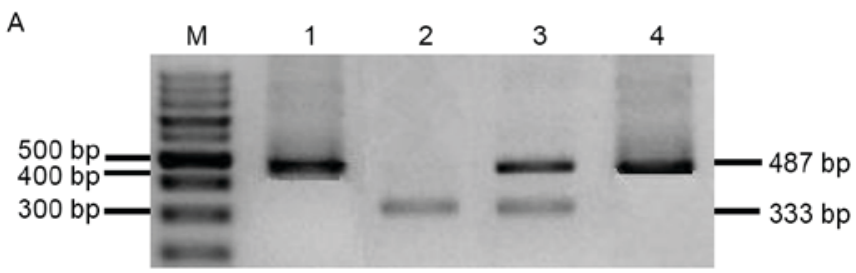

B

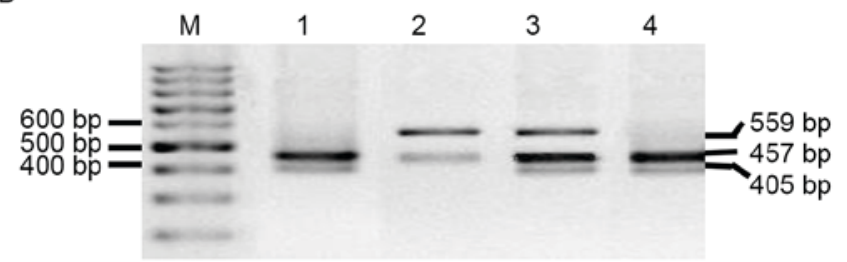

Figure 2. Electrophoresis of the inverse shifting-polymerase chain reaction products of pedigree 1 for the detection of (A) inv22, and (B) del22 or dup22. Lane M, marker; lane 1, normal male control (band size, A: 487 bp; B: 457 and 405 bp; normal); lane 2, proband (band size, A: 333 bp; B: 559 and 457 bp; inv22-1); lane 3, mother (band size, A: 487 and 333 bp; B: 559, 457 and 405 bp; inv22-1 heterozygote); lane 4, father (band size, A: 487 bp; B: 457 and 405 bp; normal). inv22, intron 22 inversion; del22, intron 22 deletion; dup22, intron 22 duplication.

the proband's mother was inherited from the proband's maternal grandmother (I2), and the normal X chromosome was inherited from the proband's maternal grandfather (I1). The proband's maternal grandmother and aunt were carriers, and the proband's maternal grandfather was normal. The band of the baby son of the proband's maternal aunt (III3) was in the same position as the upper band of the proband's maternal aunt (II5), indicating their $\mathrm{X}$ chromosome was normal. In addition, the number of STR repeats (detected via capillary electrophoresis) of DXS9901(GT) $\mathrm{n}$ and 13(CA)n loci in each sample is presented in Fig. 3C and D.

The consistency in the size of the enzyme-digested products of int18 (Fig. 3E), int19 (Fig. 3F) and int22 (Fig. 3G) loci indicated that the $\mathrm{X}$ chromosome of the proband (III), the same as one of the mother's (II3) X chromosomes, was pathogenic and thus, the mother was a carrier. The $\mathrm{X}$ chromosome of the proband's baby brother (III2) was not pathogenic, indicating he was normal. One of the $\mathrm{X}$ chromosomes of the proband's maternal grandmother (I2) was pathogenic while the $\mathrm{X}$ chromosome of the proband's maternal grandfather (I1) was normal, demonstrating that the mother's pathogenic and normal X chromosome came from the proband's maternal grandmother and maternal grandfather, respectively. The 


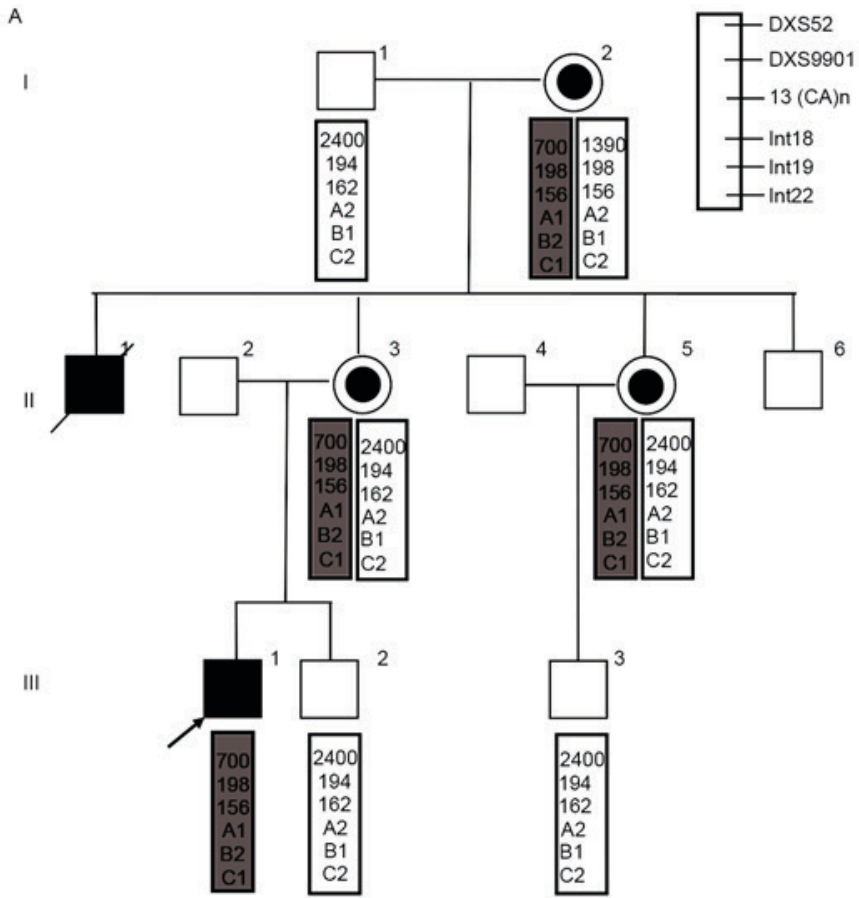

B

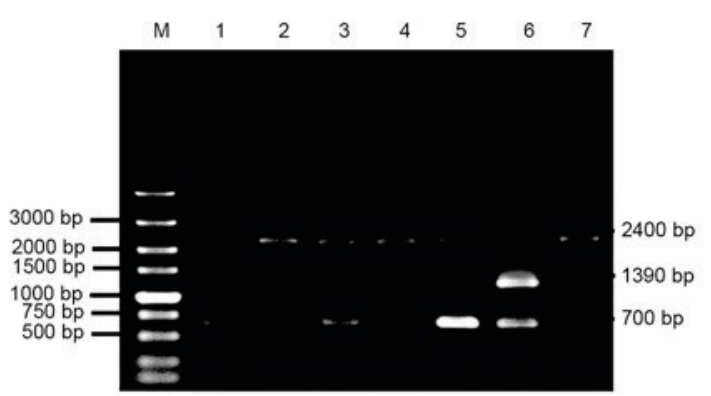

D

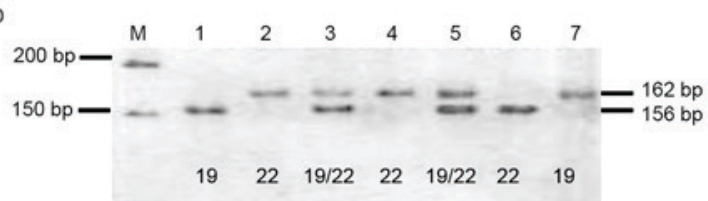

C

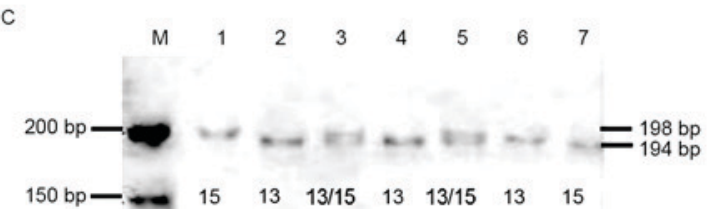

F

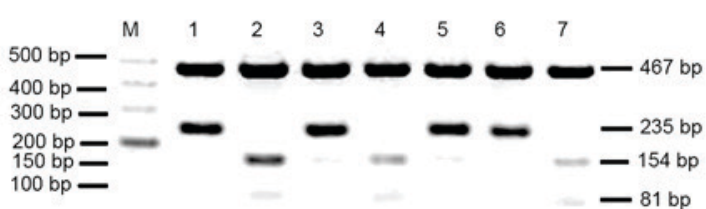

E

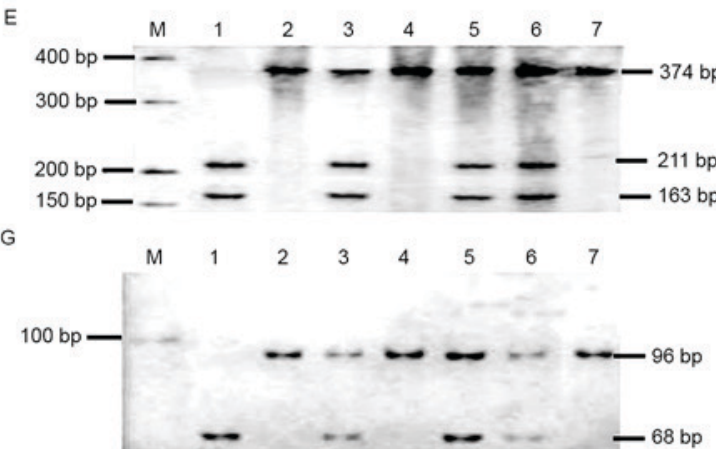

Figure 3. Genogram and electrophoretogram of the PCR products of pedigree 2. (A) The haplotype linkage analysis results of the DXS52, DXS9901(GT)n, 13(CA)n, int18, int19 and int22 genetic marker loci of pedigree 2. According to the mutation haplotype of III1 (proband; indicated by an arrow), the grey bar represents the haplotype with a mutation while the white bar represents the haplotype without a mutation, the numbers within the grey and white bars indicate fragment size (bp) from the PCR. White squares, normal males; black square with an arrow, proband; black square with a forward slash, deceased male patient; black/white circles, female carriers; A1, int18/BclI allele positive; A2, int18/BclI allele negative; B1, int19/HindIII allele positive; B2, int19/Hind III allele negative; C1, int22/XbalI allele positive; C2, int22/XbalI allele negative. Electrophoresis of PCR products for the (B) DXS52, (C) DXS9901 (GT)n, (D) 13(CA)n, (E) int18, (F) int19 and (G) int22 loci. The numbers written on the blots (C and D) represent the number of short tandem repeats and refers to the genotype of each sample. Lane M, marker; lane 1, III1; lane 2, III2; lane 3, II3; lane 4, III3; lane 5, II5; lane 6, I2; lane 7, I1. PCR, polymerase chain reaction; int, intron.

proband's maternal grandmother was a carrier and the proband's maternal grandfather was normal. One of the proband's maternal aunt's (II5) chromosomes was pathogenic and inherited from the proband's maternal grandmother, while the other normal $\mathrm{X}$ chromosome was inherited from the proband's maternal grandfather. The proband's maternal aunt was a carrier. Since her son (III3) had no pathogenic X chromosome, he could be diagnosed as normal.

To conclude the results of pedigree 2 , the pathogenic $\mathrm{X}$ chromosome of the proband was inherited from the proband's mother who was a carrier. The proband's baby brother was normal and therefore, has no risk of severe bleeding in the future. The proband's maternal grandmother was a carrier and the proband's maternal grandfather was normal. The proband's maternal aunt was a carrier, and her baby son was normal and, therefore, also has no risk of severe bleeding in the future.

Pedigree 3. Detection of 11 genetic marker loci for pedigree 3 identified four marker loci that were heterozygous and could provide effective linkage information, including one STR locus [DXS9901(GT)n] and three RFLP loci (int18, int19 and int22). The genogram and the electrophoretogram of the PCR products of pedigree 3 are presented in Fig. 4.

The results of DXS9901(GT)n locus analysis revealed that the band of the proband (III3) was in the same position as the proband's mother's (II5) lower band, indicating that the $\mathrm{X}$ chromosome was pathogenic and the mother was a carrier (Fig. 4B). One of the two bands of the proband's baby sister (III4) was from the mother (the pathogenic $\mathrm{X}$ chromosome) and the other band was from the father (II4; the normal $\mathrm{X}$ chromosome), indicating that the proband's baby sister was a carrier. There was only one band for the proband's maternal grandmother (I2), which was in the same position as the upper band (the normal X chromosome) of the proband's mother, indicating it was not pathogenic. In addition, the number of STR repeats (detected via capillary electrophoresis) of the DXS9901(GT)n locus was marked in Fig. 4B. 

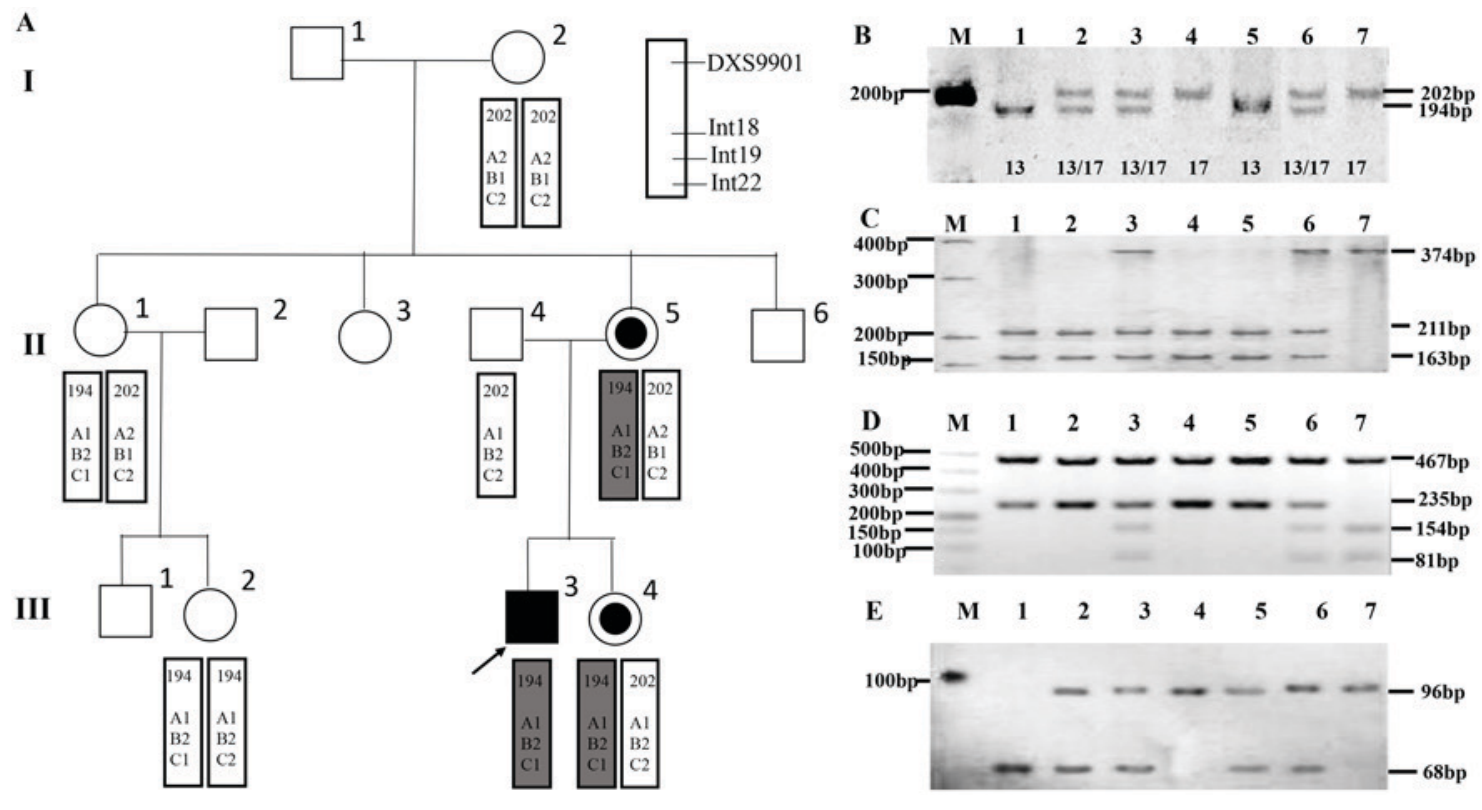

Figure 4. Genogram and electrophoretogram of the PCR products of pedigree 3. (A) The haplotype linkage analysis results of the DXS9901(GT)n, int18, int19 and int22 genetic marker loci of pedigree 3. According to the mutation haplotype of III3 (proband; indicated by an arrow), the grey bar represents the haplotype with a mutation while the white bar represents the haplotype without a mutation, the numbers within the grey and white bars indicate fragment size (bp) from the PCR. White squares, normal males; black square with an arrow, proband; black/white circles, female carriers; white circles, normal females; A1, int18/Bcl I allele positive; A2, int18/BclI allele negative; B1, int19/HindIII allele positive; B2, int19/HindIII allele negative; C1, int22/XbalI allele positive; C2, int22/XbalI allele negative. Electrophoresis of the PCR products of the (B) DXS9901(GT)n, (C) int18, (D) int19 and (E) int22 loci. The numbers written on the blots (B) represent the number of short tandem repeats and refers to the genotype of each sample. Lane M, Marker; lane 1, III3; lane 2, III4; lane 3, II5; lane 4, II4; lane 5, III2; lane 6, II1; lane 7, I2. PCR, polymerase chain reaction; int, intron.

The results of the int18 (Fig. 4C), int19 (Fig. 4D) or int22 (Fig. 4E) loci analysis demonstrated that the $\mathrm{X}$ chromosome of the proband (III3) was the same as one of the mother's (II3), as the size of enzyme-digested products were the same. Therefore, this $\mathrm{X}$ chromosome was pathogenic and the mother was a carrier. The pathogenic $\mathrm{X}$ chromosome of the proband's baby sister (III4) was inherited from the mother and the normal $\mathrm{X}$ chromosome was from the father (II4). Therefore, the baby sister was a carrier. The two X chromosomes of the proband's maternal grandmother (I2) were not pathogenic, therefore, the proband's maternal grandmother was normal.

To conclude the results of pedigree 3, the proband's maternal grandmother was normal and the pathogenic $\mathrm{X}$ chromosome of the proband was inherited from the proband's maternal grandfather. As the proband's grandfather was not diagnosed with HA, it is possible that a mutation of the FVIII gene occurred in the germ cell of the maternal grandfather, which was passed on to the mother. As with the proband's mother, the baby sister of the proband was also a carrier, and therefore, if she were to have sons they would be at risk of HA. However, the $\mathrm{X}$ chromosomes of the proband's maternal uncle and aunts, though inherited from the maternal grandfather, did not have the same mutant as the proband's mother, indicating that the maternal aunts and their female offspring were not carriers. The maternal uncle was normal.

\section{Discussion}

A total of $\sim 40 \%$ of severe cases of HA have a genetic etiology of inv22 of the FVIII gene (12), whereas inv1 is present in only $2-5 \%$ of severe cases $(13,14)$. Hence, a direct method should be performed first to detect inversion mutations for patients with severe HA. Long distance PCR was once advocated to detect inv22; however, its limitations, including difficulties in amplification, a stringent requirement for template quality and poor repeatability, make it less reliable (15). In recent years, investigators began to use inversion PCR (I-PCR), which consists of enzyme digestion, T4 ligase connection and PCR amplification. The advantages of I-PCR include lower quality requirement for templates, shorter DNA fragments to be amplified, good repeatability, an easy protocol and simple interpretation of the results (15). This research adopted the IS-PCR method (7), which was the result of optimization and improvements made to the I-PCR protocol. IS-PCR can detect either inv22 or inv1, and homozygous patients or heterozygous carriers, it can distinguish inv22 distal inversion with proximal inversion, and it can eliminate the possibility of misdiagnosing del22 or dup22 as inv22. Previous studies including the use of the IS-PCR method for detecting HA have been infrequent until recently, and IS-PCR may be widely applied in clinical direct gene diagnosis for HA (16).

The FVIII gene is associated with significant genetic heterogeneity, a large structure and complex alterations to molecular pathology, thus, current molecular biology methods have not been able to uncover all of the molecular defects (2). This renders the direct gene diagnosis of HA challenging (in all cases except for inv22). Consequently, the use of genetic markers inside or nearby the FVIII gene for indirect genetic diagnosis has become one of the main methods for HA gene diagnosis (17).

Indirect genetic diagnosis for HA generally uses genetic markers, including VNTR, STR and RFLP, for linkage analysis. The commonly-used extragenic VNTR or STR loci include DXS52 (18,19), DXS15(CA)n (18,20), DXS9901(GT)n 
and DXS1073(GT)n (10). Intragenic STR loci include 13 (CA)n $(11,21,22)$ and $22(\mathrm{GT}) \mathrm{n}(\mathrm{AG}) \mathrm{n}(21,22)$. Intragenic RFLP loci include BclI (23,24), HindIII (23,24), XbalI (23) and BglI (18) enzyme cutting loci in introns 18, 19, 22 and 25, respectively. Genetic markers used for linkage analysis should have a certain rate of heterozygosis (usually at least 30\%) and should provide sufficient polymorphism information in a certain population. In general, in accordance with the heterozygous rate of the local population, genetic markers with a high heterozygous rate should be selected, and the combine use of several genetic markers may improve the efficiency of diagnosis. According to a previous report, the heterozygous rate of the RFLP loci int18, int19 and int22 in the north Indian population was 57, 38 and $43 \%$, respectively (23). The heterozygous rate of the STR loci in the Chinese population have been reported to be $34.6 \%$ (11) or $37.9 \%$ (22) for intron 1 (GT)n, $60.9 \%(25)$ or $61.0 \%(11,22)$ for $13(\mathrm{CA}) \mathrm{n}, 43.6 \%$ (11) for $22(\mathrm{GT}) \mathrm{n}(\mathrm{AG}) \mathrm{n}$ and $38.2 \%$ (11) for 24(GT)n, respectively. The heterozygous rate of extragenic genetic markers of the STR loci DXS15(CA)n, DXS9901(GT)n and DXS1073(GT)n in the Chinese population have been reported to be $88.24(10), 82.35(10)$ and $62.0 \%$ (25), respectively. Therefore, the present study applied these 11 genetic marker loci with high heterozygous rates for indirect genetic diagnosis in Chinese pedigrees. As there is a $3 \%$ recombination possibility within the large HA gene during meiosis (10), the present study used multiple heterozygous loci for haplotype linkage analysis in order to avoid incorrect linkage analysis as result of recombination between the genetic marker locus and the mutation locus.

In conclusion, the results of the present study may have academic and clinical implications. Linkage analysis could be used to diagnose affected pedigree members other than the proband, particularly presymptomatic males who would suffer from severe bleeding following trauma. Linkage analysis could also be used to accurately diagnose female carriers from potential female carriers. On this basis, linkage analysis could be applied for prenatal diagnosis in the male infants of female carriers, including all accurately diagnosed female carriers within the pedigree, not just the patient's mother. The results of the present study indicated that the combination of direct and indirect gene diagnosis may provide reliable evidence for the use of genetic counseling for HA pedigrees, particularly for screening presymptomatic males and female carriers with normal or no offspring, which may facilitate earlier treatments and disease prevention.

\section{References}

1. Harraway JR, Smith MP and George PM: A highly informative, multiplexed assay for the indirect detection of hemophilia A using five-linked microsatellites. J Thromb Haemost 4: 587-590, 2006.

2. Kim JW, Park SY, Kim YM, Kim JM, Kim DJ and Ryu HM: Identification of new dinucleotide-repeat polymorphisms in factor VIII gene using fluorescent PCR. Haemophilia 11: 38-42, 2005.

3. Keeney S, Mitchell M and Goodeve A; UK Haemophilia Center Doctors' Organization Haemophilia Genetics Laboratory Network: The molecular analysis of haemophilia A: A guideline from the UK haemophilia centre doctors' organization haemophilia genetics laboratory network. Haemophilia 11: 387-397, 2005.
4. Li W, Hu X, Gao BD, Li LY, Liao Y, Tang XM, Tang WL and Lu GX: Mutation screening of the FVIII gene in 10 hemophilia A families. Zhonghua yi xue yi Chuan xue za zhi 28: 127-132, 2011 (In Chinese).

5. Pandey GS and Mittal B: Molecular diagnosis in haemophilia A. J Postgrad Med 47: 274-280, 2001.

6. Köchl S, Niederstätter $\mathrm{H}$ and Parson W: DNA extraction and quantitation of forensic samples using the phenol-chloroform method and real-time PCR. Methods Mol Biol 297: 13-30, 2005.

7. Rossetti LC, Radic CP, Larripa IB and De Brasi CD: Developing a new generation of tests for genotyping hemophilia-causative rearrangements involving int $22 \mathrm{~h}$ and int $1 \mathrm{~h}$ hotspots in the factor VIII gene. J Thromb Haemost 6: 830-836, 2008.

8. Yan M, Liang Y, Chen X, Fan XP, Xiao B and Liu JZ: Improvement and application of DXS52 (St14) in gene diagnosis of hemophilia A. Zhonghua Yi Xue Yi Chuan Xue Za Zhi 28: 19-22, 2011 (In Chinese).

9. Richards B, Heilig R, Oberlé I, Storjohann L and Horn GT: Rapid PCR analysis of the St14 (DXS52) VNTR. Nucleic Acids Res 19: 1944, 1991.

10. Fang Y, Wang XF, Dai J and Wang HL: A rapid multi-fluorescent polymerase chain reaction for genetic counseling in Chinese haemophilia A families. Haemophilia 12: 62-67, 2006.

11. Liang Y, Zhao Y, Yan M, Fan XP, Xiao B and Liu JZ: Prenataldiagnosis of haemophilia A in China. Prenat Diagn 29: 664-667, 2009.

12. Lakich D, Kazazian HH Jr, Antonarakis SE and Gitschier J: Inversions disrupting the factor VIII gene are a common cause of severe haemophilia A. Nat Genet 5: 236-241, 1993.

13. Gouw SC, van den Berg HM, Oldenburg J, Astermark J, de Groot PG, Margaglione M, Thompson AR, van Heerde W, Boekhorst J, Miller CH, et al: F8 gene mutation type and inhibitor development in patients with severe hemophilia A: Systematic review and meta-analysis. Blood 119: 2922-2934, 2012.

14. Rossetti LC, Radic CP, Larripa IB and De Brasi CD: Genotyping the hemophilia inversion hptspot by use of inverse PCR. Clin Chem 51: 1154-1158, 2005.

15. Kumar P, Faridi NJ, Husain N, Soni P and Goel SK: Study of intron 22 inversion mutation in north India with review. Blood Coagul Fibrinolysis 24: 120-124, 2013.

16. Abou-Elew H, Ahmed H, Raslan H, Abdelwahab M, Hammoud R, Mokhtar D and Arnaout H: Genotyping of intron 22: Related rearrangement of F8 by inverse-shifting PCR in egyptian hemophilia A patients. Ann Hematol 90: 579-584, 2011.

17. Chowdhury MR, Tiwari M, Kabra M and Menon PS: Prenatal diagnosis in hemophilia A using factor VIII gene polymorphism-indian experience. Ann Hematol 82: 427-430, 2003.

18. Lalloz MR, Schwaab R, Mcvey JH, Michaelides K and Tuddenham EG: Haemophilia A diagnosis by simultaneous analysis of two variable dinucleotide tandem repeats within the factor VIII gene. Br J Haematol 86: 804-809, 1994.

19. Liu Y, Wang X, Chu H, Li Z, Wang H and Wang Z: Carrier detection and prenatal diagnosis of hemophilia Alpha. Chin Med J (Engl) 115: 991-994, 2002.

20. He Z, Chen J, Xu S, Chen S, Xiao X, Li H, Guo Y and Jiang W: A strategy for the molecular diagnosis in hemophilia a in Chinese population. Cell Biochem Biophys 65: 463-472, 2013.

21. Machado FB and Medina-Acosta E: High-resolution combined linkage physical map of short tandem repeat loci on human chromosome band Xq28 for indirect haemophilia A carrier detection. Haemophilia 15: 297-308, 2009.

22. Massaro JD, Wiezel CE, Muniz YC, Rego EM, de Oliveira LC, Mendes-Junior CT and Simões AL: Analysis of five polymorphic DNA markers for indirect genetic diagnosis of haemophilia A in the Brazilian population. Haemophilia 17: e936-e943, 2011.

23. Tasleem Raza S, Husain N and Kumar A: Screening for hemophilia A carriers: Utility of PCR-RFLP-based polymorphism analysis. Clin Appl Thromb Hemost 15: 73-83, 2009.

24. Moharrami T, Derakhshan SM, Pourfeizi AA and Khaniani MS: Detection of hemophilia A carriers in azeri turkish population of Iran: Usefulness of HindIII and Bcli markers. Clin Appl Thromb Hemost 21: 755-759, 2015.

25. Ding QL, Lu YL, Dai J, Xi XD, Wang XF and Wang HL: Characterisation and validation of a novel panel of the six short tandem repeats for genetic counseling in Chinese haemophilia A pedigrees. Haemophilia 18: 621-625, 2012. 